\title{
Fatigue system reliability analysis of riveted railway bridge connections
}

\author{
Boulent M. Imam ${ }^{\mathrm{a}^{*}}$; Marios K. Chryssanthopoulos ${ }^{\mathrm{a}}$; Dan M. Frangopol ${ }^{\mathrm{b}}$ \\ ${ }^{a}$ Faculty of Engineering and Physical Sciences, University of Surrey, Guildford, \\ Surrey GU2 $7 X H, U K$ \\ ${ }^{b}$ Department of Civil and Environmental Engineering, Engineering Research Center \\ for Advanced Technology for Large Structural Systems (ATLSS), Lehigh University, \\ Bethlehem, PA 18015-4729, USA \\ *Corresponding author. Email: b.imam@surrey.ac.uk
}

\begin{abstract}
A system-based model for fatigue assessment of riveted railway bridge connections, comprising a number of basic components, is presented in this paper. Probabilistic fatigue load spectra are developed through Monte Carlo simulation of train passages over a finite element model of a typical, short-span bridge. Uncertainties arising from loading, resistance and modelling sources are taken into account. The riveted connection is treated through a set of generic sub-systems that capture potential damage in identifiable hotspots, such as rivets, holes and angle fillets. The fatigue reliability over time is evaluated through system reliability methods by treating these hot-spots as the elements of a structural system. The results show that the probability of failure of the connection depends significantly on the form of the system adopted for the analysis and the rivet clamping force. Damage scenarios accounting for the potential loss of rivet clamping force are investigated and it is shown that, in some cases, they can affect connection reliability considerably.
\end{abstract}

Keywords: structural systems; bridges; reliability; fatigue; time; damage

\section{Introduction}

Fatigue assessment of riveted bridges has received considerable attention during the past few decades. This is attributed to the fact that such bridges, which were not designed explicitly for fatigue, are believed to be approaching the end of their service life. Considering their large number, both in Europe and North America, replacement of all such bridges is extremely challenging from an economic point of view and would create severe network problems. In order to maintain these old bridges and plan repairs effectively, assessment of their remaining fatigue life is a vital requirement.

Riveted bridges were constructed using built-up sections which comprise several typically flat plated elements riveted together. The primary bridge members, such as girders, stringers and floor-beams are joined through riveted connections 
consisting of a number of rivets and angle clips. The redundancy of these structures is generally high, since failure of an individual component does not usually lead to progressive collapse. This is because in a typical riveted member, a fatigue crack will propagate only within the component that has cracked and will not immediately transfer to other adjoining components since the interfaces between components act as crack stoppers (Sweeney 1979). However, following failure of the first component and redistribution of the loads to the remaining components, a progressive type of failure may be initiated, depending on geometric configuration, acting load levels and component utilisation. It is, hence, both rational and desirable to extend methods to estimate the reliability of riveted bridges and connections beyond the level of individual component failure towards a systems reliability framework, which captures the relationships between individual component and overall system performance.

One of the appealing features of using a system approach to reliability is that it considers both the reliability of components and their relationship and importance to the entire system, thus allowing quantification of redundancy. In some cases, when a system is highly redundant, the reliability of individual components may be quite low while the system reliability remains high. In other cases, for weakest-link systems, although the reliability of every component may be satisfactory, the reliability of the system might be considered low. A system analysis is capable of capturing and quantifying such effects. On the other hand, considering individual components alone, which is still a common practice, may underestimate or overestimate the overall reliability of a structure. A number of recent studies have investigated the redundancy of structural systems from a system reliability point of view (Ghosn et al. 2010, Okasha and Frangopol 2010). 
System reliability approaches have been commonly used in the past to estimate the service life of bridge structures (Hendawi and Frangopol 1994, Micic et al. 1995, Estes and Frangopol 1999, Nowak and Cho 2007). The deck, superstructure and substructure can be broken down to a varying degree and modelled as components of a higher level system representing the bridge. The reliability of different component failure modes can be estimated using methods such as FORM (First Order Reliability Method), SORM (Second Order Reliability Method) or through Monte Carlo simulations. A system representing the bridge is then assembled as a series-parallel combination of its components and a system approach is employed to estimate the overall system reliability (Melchers, 1999).

System reliability analysis of riveted bridges and connections has received limited attention, with only a few recent studies in that area. Wang et al. $(2006,2007)$ developed a system fatigue damage reliability model based on fracture mechanics principles investigating the behaviour of a riveted built-up girder. Probabilistic crack growth analysis of cracks initiating from the holes of angle clips were carried out and combined through systems analysis to estimate the reliability of the riveted girder. As a case study, their model was used to determine safe inspection intervals and maintenance strategies for an existing riveted bridge in China.

The objective of this paper is to present a system-based model for fatigue assessment of riveted bridge connections building on previous work which has quantified a number of important features exhibited by riveted grillage-type structures (Imam et al. 2006, Imam et al. 2008, Righiniotis et al. 2008). A finite element (FE) model of a short span, wrought iron bridge is used in order to convert train loading into fatigue load effects. Fatigue assessment is carried out using the recently developed theory of critical distances (TCD) which considers the entire distribution 
ahead of any given stress concentration. This theory (Taylor 2007), allows the criticality of different parts of a fatigue sensitive detail to be independently assessed, and lends itself to a system-based treatment of fatigue failure. A system approach is presented by treating each riveted connection through generic sub-systems which capture potential damage in identifiable hot-spots, such as rivets, holes and angle fillets. By treating these hot spots as the elements of a structural system susceptible to fatigue failure, its reliability over time is evaluated using system reliability methods. The relative importance of various connection components, given their individual failure probability and their contribution to the system reliability, is discussed. The effect of different damage scenarios, in the form of loss of rivet clamping force, on the overall system reliability of the connection is also investigated through parametric studies. The results are compared with their S-N counterparts, which were obtained by the authors in earlier studies (Imam et al. 2008) by employing the traditional nominal stress approach, commonly adopted in bridge codes (BS5400 1980, AREA 1996), and modelling the connection as a single component.

\section{Fatigue Assessment using the Theory of Critical Distances}

The most popular method of fatigue analysis of structural components and connections is the well-known S-N method which is employed by the majority of bridge codes (AASHTO 1998, AREA 1996, BS5400 1980, EC3 2005). The S-N method requires the classification of a detail into a fatigue class among typical standardised "basic details" and its fatigue damage is estimated using the relevant S-N curve combined with remotely applied nominal stresses. Despite the fact that existing standards and recommendations cover a wide range of structural details and loading situations, there are a number of complex details that cannot be clearly classified or 
even if they can, the definition of nominal stresses for these is far from straightforward. One such example is deformation-induced fatigue which is caused by secondary stresses. In riveted bridges, such type of fatigue damage may take place at riveted connections such as, for example, stringer-to-cross-girder connections ( $\mathrm{Al}$ Emrani 2002). Conventional methods for fatigue analysis such as the S-N nominal stress method have in many cases proven themselves to be inappropriate for deformation-induced fatigue (Imam 2006). Assessment of existing bridges with such details using the conventional methods may render unrealistic results which can make a correct condition assessment and planning of maintenance strategies more difficult.

During the last decade or so, alternative fatigue design and assessment methods have been developed such as the geometric (or hot spot) stress approach (DNV 2005), the structural stress approach (Dong 2001), and the Theory of Critical Distances (TCD) (Taylor 2007). The hot spot stress approach has been widely used in the offshore industry and the TCD has been commonly employed in fatigue assessment of mechanical and automotive components. All of these methods are based on linear elastic finite element (FE) analysis and can be used for fatigue assessment of complex details for which the S-N method is difficult to utilise. However, these approaches have so far rarely been used by bridge engineers and need to be developed and adapted for bridge assessment applications.

The hot spot (or geometric) stress approach is based on extrapolation of stresses obtained at a distance from the stress concentration (notch) of the fatigue detail being investigated. On the other hand, the structural stress method is based on defining an equivalent stress state at some distance from the stress concentration and considered for fatigue calculations. Both of these methods have been found to give reasonable results for two-dimensional (2D) welded details but have been shown to 
have certain limitations for three-dimensional (3D) details and are difficult to apply in cases of complex 3D loadings (Doerk et al. 2003). In additional, their suitability for the fatigue assessment of non-welded details, such as riveted connections, has not been established so far.

The TCD is a recently developed methodology for fatigue assessment of notched components (Taylor 2007), in which the entire stress distribution ahead of the notch is considered for fatigue calculations. In other words, TCD does not rely on a single, remotely applied stress, as postulated by the S-N approach, which may be difficult to define, or on a stress at a single stress concentration point. This theory, which can be applied to any type of stress concentration, was developed so as to be used in conjunction with linear elastic FE analyses. These have advanced extensively during recent years and allow analyses of complex, 3D geometries and reasonably accurate predictions of stress fields in the vicinity of stress concentrations.

One of the main advantages of the TCD is that it requires physical characterisation of the plain material only, rather than the extensive, but often lacking, physical testing on specific detail geometries. A fundamental aspect of the TCD is to determine a location or region within which all physical processes leading to fatigue crack initiation are assumed to take place. This size/location (or region) depends on fatigue material properties and more specifically on the fatigue limit of the plain material and its crack propagation threshold (threshold stress intensity factor). All stress calculations required for fatigue assessment are carried out within this region, the size/location of which can be characterised through a "critical distance", $L$, defined as (Taylor 1999)

$$
L=\frac{1}{\pi}\left(\frac{\Delta K_{t h}}{\Delta \sigma_{0}}\right)^{2}
$$


where $\Delta K_{t h}$ is the threshold stress intensity (crack propagation threshold) and $\Delta \sigma_{0}$ is the fatigue limit of the plain (un-notched) material.

Following the definition of the critical distance $L$, there are different methods that may be employed for fatigue assessment based on this parameter. The point method suggests considering the maximum principal stress $\sigma_{p(\max )}$ at a distance of $L / 2$ from the tip of the notch (Taylor 1999). The line method suggests averaging the maximum principal stress along a line of length $2 L$ starting from the notch tip (Taylor 1999). In the area method, the maximum principal stress is averaged over a semicircular area, which is centred at the notch tip, having a radius of $1.32 L$ (Bellett et al. 2005). For $3 \mathrm{D}$ applications, the volume method, which relies on averaging principal stresses over a hemisphere centred at the tip of the notch with a radius of $1.54 \mathrm{~L}$ has been proposed (Bellett et al. 2005). After determining the "characteristic stress" by one of these methods, this is then used for fatigue damage calculations.

Although the point and line methods may appear less complicated, analysis using the area and volume methods is not difficult to implement as part of postprocessing after an FE analysis. The volume method lends itself to the analysis of 3D geometries where there are 3D stress fields around the investigated stress concentration since the method can capture stress gradients in all directions.

The reliability of the TCD for fatigue assessment has been verified through comparisons of analytical and experimental results on a wide range of stress concentrations and materials where it was shown that it can predict accurately both fatigue strength and fatigue life (Taylor 1999, 2005, Taylor and Wang 2000, Susmel and Taylor 2003).

A further appealing feature of the TCD is that it overcomes the mesh sensitivity problem that exists in FE analysis of stress concentrations where, in 
general, the maximum stress at notches does not converge with increasing mesh density since these points are usually associated with numerical singularities. As a result, the fatigue damage becomes unbounded. Since the TCD relies on the entire stress distribution ahead of the stress concentration and not on a single maximum stress value, it can overcome this problem. Previous studies carried out on a typical riveted bridge connection model have demonstrated the convergence characteristics of the TCD volume method (Righiniotis et al. 2008).

\section{Reliability Analysis Framework}

\subsection{Finite element analysis}

A probabilistic methodology has been employed in earlier studies by the authors for the fatigue assessment of a typical wrought iron riveted railway bridge (Imam et al. 2008). It is based on the S-N nominal stress method and accounts for uncertainties in loading, resistance and modelling. A global FE model of the bridge (shown in Figure 1) was used to convert present-day and historical rail traffic into fatigue load effects. Annual fatigue response spectra were developed for different periods of the traffic models, spanning over 100 years, and were used for the estimation of the fatigue reliability of a stringer-to-cross-girder connection over time.

As the global model of the bridge treated the connection as a single entity and was unable to capture the fatigue behaviour of its individual elements, a detailed submodel of the connection was later developed within the global model. This model, which has been fully described and validated in previous studies (Imam et al. 2007), is shown in Figures 2 and 3. As can be seen, all the individual elements of the connection such as angle clips and rivets and parts of the connected members in the vicinity of the connection were modelled explicitly to investigate their fatigue behaviour and assess their fatigue criticality. Fatigue damage calculations, which were 
carried out deterministically, were based on the TCD volume method and the fatigue critical hot spots on the connection were found to be located around the perimeter of the holes, around the circumference of the rivet head-to-shank intersection and along the depth of the angle fillet (Righiniotis et al. 2008).

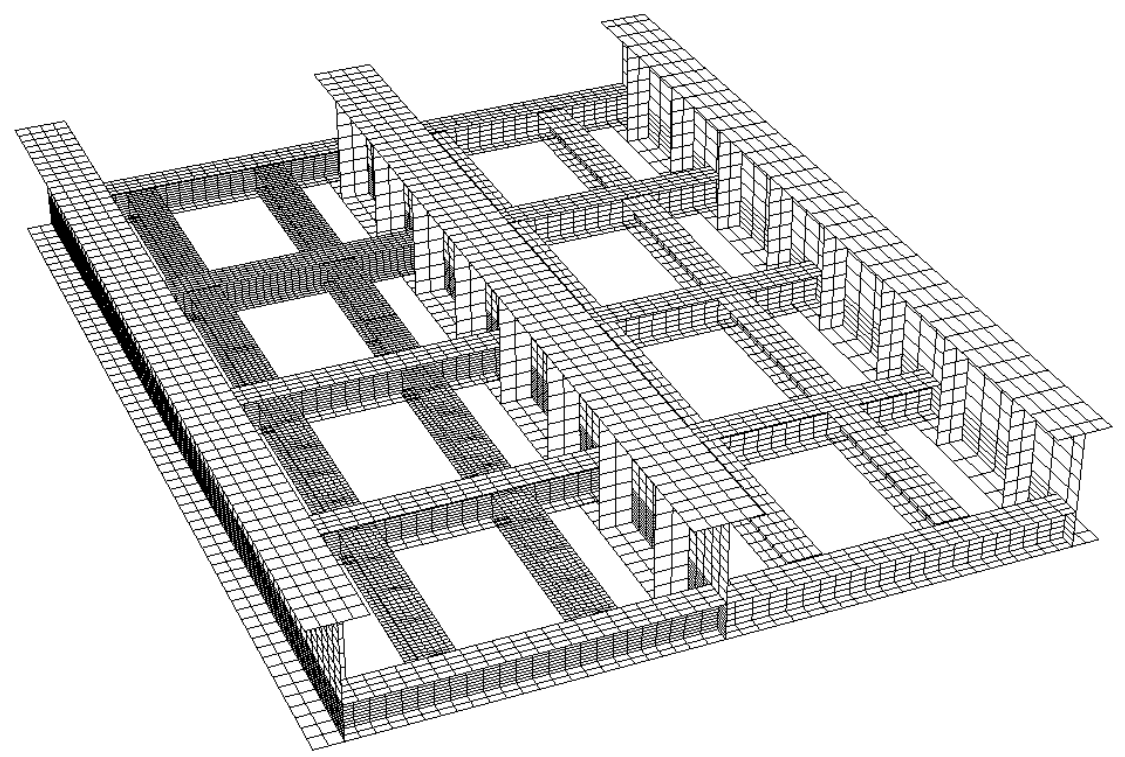

Figure 1. Global FE model of the investigated riveted bridge (Imam et al. 2007).

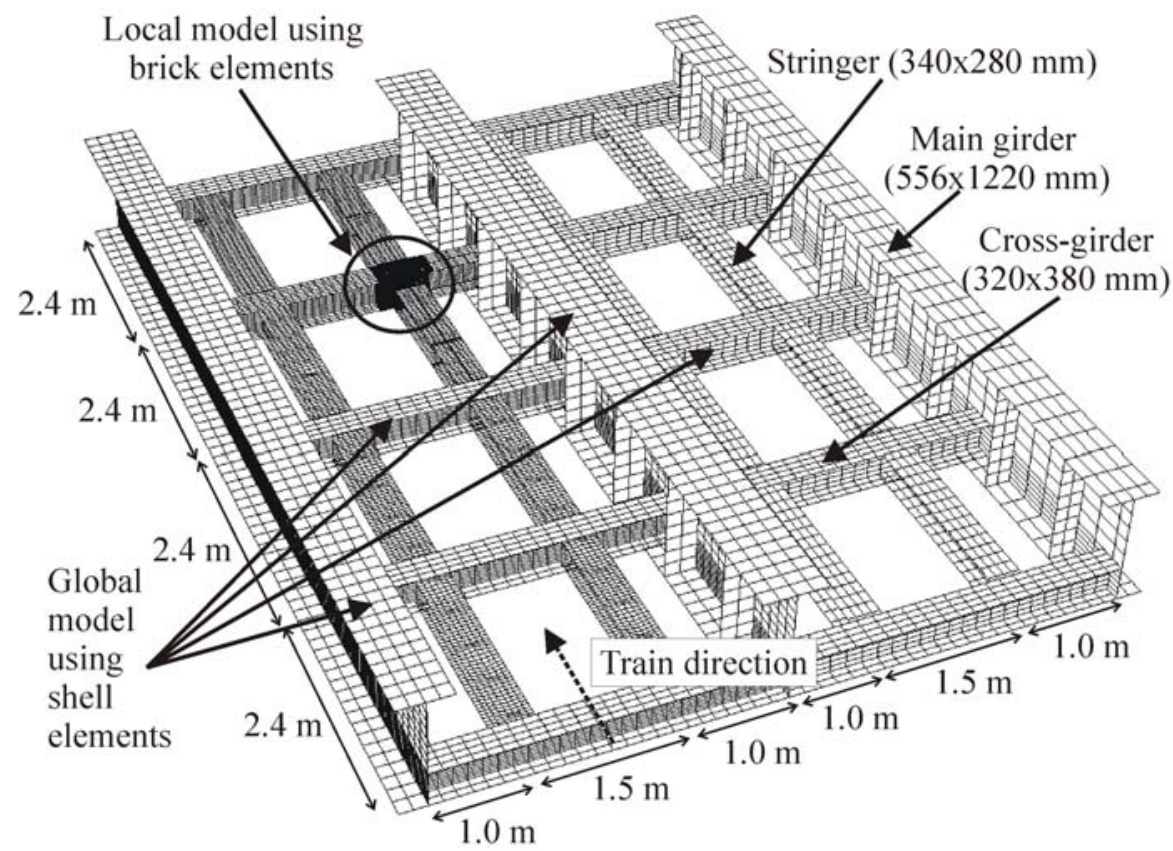

Figure 2. Refined FE model of the investigated riveted bridge (Imam et al. 2007). 


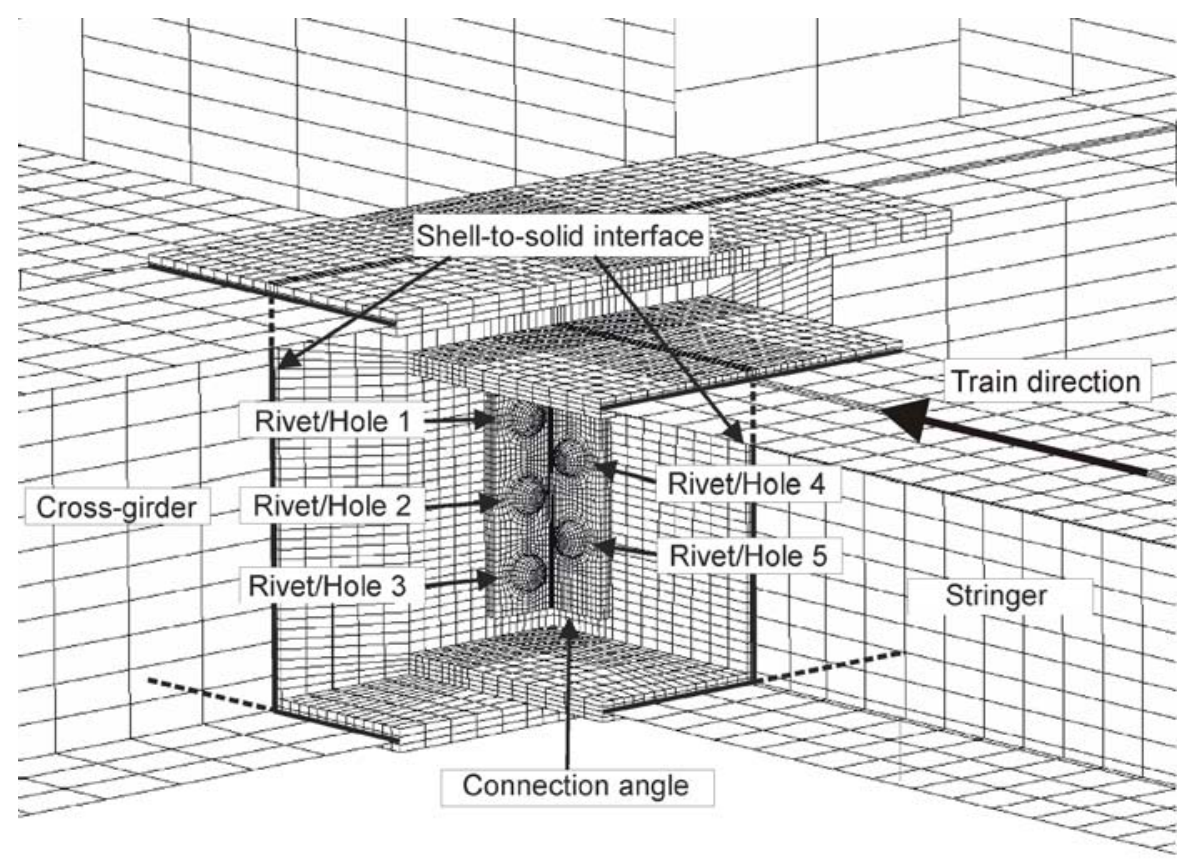

Figure 3. Close-up view of the detailed connection FE model indicated in Figure 2.

In this paper, the previous deterministic fatigue analyses using the TCD method (Righiniotis et al. 2008) are extended within a probabilistic framework. To this end, principal stress histories are obtained in the vicinity of the critical hot spots of the connection (i.e. holes, rivets, angle clip fillets) by traversing trains over the refined FE model of the bridge. The histories are obtained at the elements located within the critical volumes which are in the form of a hemisphere centred at the notch and having a radius of $1.54 \mathrm{~L}$. These stress histories are then combined in the context of the TCD volume method to obtain the characteristic (averaged within the volume) stress histories at each hot spot (stress concentration) which are used with the rainflow counting method and Miner's rule (Miner 1945) to calculate fatigue damage. Uncertainties in loading, resistance and modelling are introduced at this stage. 


\subsection{Random variables}

\subsubsection{Resistance random variables}

On the resistance side, uncertainties in the S-N curves and the cumulative damage model (Miner's rule) are incorporated within the methodology. For fatigue damage calculations, the S-N curve is treated probabilistically by assuming a fixed slope and a random fatigue limit. For wrought iron, a lognormal distribution with a mean value of $183 \mathrm{MPa}$ defined at $N_{F L}=2 \times 10^{6}$ cycles and a $\mathrm{CoV}$ of 0.1 is assumed for the fatigue limit $\Delta \sigma_{0}$ (Righiniotis et al. 2008) together with a slope of $1 / 5$ (Cullimore 1967). In graphical terms, the effect of random variability of the fatigue limit is to produce a parallel shifting of the S-N curve with the fatigue limit always defined at $2 \times 10^{6}$ cycles.

The damage limit $\Delta$ in Miner's sum is also assumed to be a random variable. In deterministic analysis, this damage limit, which indicates fatigue failure, is assumed equal to one. Herein, a lognormal distribution with a mean value of 0.90 and a CoV of 0.30 is considered in accordance with earlier studies (Wirsching 1995).

The critical distance $L$, which is used to define the critical volume for carrying out the stress calculations for fatigue assessment, has been taken as deterministic due to the large amount of FE post-processing required by the TCD method. By using the mean value for the fatigue limit of wrought iron $\left(\Delta \sigma_{0}=183 \mathrm{MPa}\right)$ and a threshold stress intensity value of $\Delta K_{t h}=13.5 \mathrm{MPa} \cdot \mathrm{m}^{1 / 2}$, the critical distance value is obtained as $L=1.73 \mathrm{~mm}$. The value of the threshold stress intensity (crack propagation threshold) has been determined through crack growth experiments on wrought iron specimens reported by Helmerich et al. (2007).

\subsubsection{Loading random variables}

On the loading side, the problem is randomised by introducing uncertainties in train traffic volume and dynamic amplification. Live loading on the bridge is represented 
by the BS5400 (1980) medium traffic model, which is also adopted in the UK for present rail traffic. The annual frequency $f_{t j}$ for each train type $j$ crossing the bridge is obtained from BS5400 and is assumed to be lognormally distributed with a coefficient of variation $(\mathrm{CoV})$ of 0.14 (Imam et al. 2008). Randomness in train frequencies is, of course, directly linked to the annual number of applied stress cycles. For the dynamic amplification factor $(D A F)$, a normal distribution with a mean value of 1.10 and a standard deviation of 0.15 is assumed. The latter is based on field measurements (Byers 1970, Tobias and Foutch 1997) on short-span steel railway bridges.

Further uncertainty in loading is incorporated by considering the epistemic or modelling uncertainty, represented by the ratio of actual-to-calculated stresses.

Modelling uncertainty is here captured by the ratio of actual-to-calculated stresses, $\alpha$, which is assumed to follow a normal distribution with a mean value of 0.80 and $\mathrm{CoV}$ of 0.14 . These values are based on comparison of stresses obtained analytically or numerically with field measurements carried out in steel railway bridges (Byers 1976, Adamson and Kulak 1995, Sweeney et al. 1997).

A summary of all the random variables and their probabilistic modelling characteristics is shown in Table 1 whereas Table 2 presents the deterministic variables used in the analyses.

\begin{tabular}{|c|c|c|c|c|}
\hline $\begin{array}{c}\text { Random } \\
\text { variable }\end{array}$ & Distribution & Mean & $\mathrm{CoV}$ & Description \\
\hline$\Delta \sigma_{0}$ & Lognormal & $183 \mathrm{MPa}$ & 0.10 & Fatigue limit \\
\hline$\Delta$ & Lognormal & 0.90 & 0.30 & Damage limit in Miner's sum \\
\hline$D A F$ & Normal & 1.10 & 0.14 & Dynamic amplification factor \\
\hline$\alpha$ & Normal & 0.80 & 0.14 & $\begin{array}{c}\text { Ratio of actual-to-calculated } \\
\text { stresses (modelling uncertainty) }\end{array}$ \\
\hline$f_{t j}$ & Lognormal & $\begin{array}{c}\text { From BS5950 } \\
(1980)\end{array}$ & 0.14 & Annual frequency for train $j$ \\
\hline
\end{tabular}

Table 1. Characteristics of random variables for reliability analysis. 


\begin{tabular}{|c|c|c|}
\hline Random variable & Mean & Description \\
\hline$\Delta K_{\text {th }}$ & $13.5 \mathrm{MPa} \cdot \mathrm{m}^{1 / 2}$ & Threshold stress intensity \\
\hline$L$ & $1.73 \mathrm{~mm}$ & Critical distance \\
\hline$m$ & 3 & Slope of S-N curve \\
\hline
\end{tabular}

Table 2. Characteristics of deterministic variables for reliability analysis.

\subsection{Fatigue reliability calculations}

The first step on the estimation of fatigue reliability is the development of annual fatigue load spectra (stress range histograms) for each critical hot-spot on the connection which will subsequently be used for fatigue damage calculations. These spectra are developed by multiplying the static stress ranges (determined from the averaged, within the volume, maximum principal stress histories) obtained from previous deterministic analyses of the bridge (Righiniotis et al. 2008) with the $D A F$ and the $\alpha$ factors. For each individual train crossing, a different value of $D A F$ and $\alpha$ is sampled from their probability distributions in order to account for randomness. This process of calculating the stress ranges is carried out $f_{t j}$ times, where $f_{t j}$ is the annual frequency of train type $j$. In effect, a Monte Carlo simulation is carried out, with random variables $D A F$ and $\alpha$ sampled from assumed distributions. Since the train frequencies $f_{t j}$ are also taken to be random, the above process is repeated $10^{3}$ times, in order to capture the uncertainty in the train frequencies.

The fatigue damage in each hot-spot is then calculated from its annual fatigue load spectrum (stress range histogram). The annual damage is given as

$$
D_{a}=\frac{1}{N_{F L}\left(\Delta \sigma_{0}\right)^{5}} \sum_{i}^{k} n_{i}\left[\left(\Delta \sigma_{\text {ave }}\right)_{i}\right]^{5}
$$

where $k$ is the number of stress range blocks in the fatigue load spectrum, $\left(\Delta \sigma_{\text {ave }}\right)_{i}$ is the $i^{\text {th }}$ stress range and $n_{i}$ is the corresponding number of applied cycles. It should be recalled that $\left(\Delta \sigma_{\text {ave }}\right)_{i}$ are the stress ranges obtained from the characteristic stress history which is calculated by averaging the maximum principal stress histories 
within the critical volume. Since train loading is of variable amplitude, in fatigue damage calculations through Equation (2), the $\mathrm{S}-\mathrm{N}$ curve is extended below $\Delta \sigma_{0}$ with the same slope $(1 / 5)$.

The probability of failure is calculated by summing up the annual damages $\left(D_{a, l}\right)$ over the years, where $l$ represents any one year, and considering a limit state function given as

$$
g=\Delta-\sum_{l}^{T} D_{a, l}
$$

where $T$ is the number of years after 1970 . The reason for performing the fatigue damage calculation from 1970 onwards is that up to that period fatigue damage was quite small, as demonstrated in earlier studies (Imam et al. 2006). For remaining fatigue life calculations, future load evolution is ignored and the BS5400 medium traffic is extrapolated into the future up to the point of fatigue failure $(g \leq 0)$. Thus, the remaining fatigue life $T_{r}$ of any hot-spot is given as

$$
T_{r}=\frac{\Delta-\sum_{l}^{T} D_{a, l}}{D_{a}}
$$

In Equation (4), variable $D_{a}$ appearing in the denominator is the mean value of the annual damage which is calculated from the annual fatigue load spectrum. For each Monte Carlo cycle, a single $\mathrm{D}_{\mathrm{a}}$ value is generated and used.

Based on the limit state function of Equation (3), the probability of fatigue failure in any one year can be defined as

$$
P_{f}=P[g(X) \leq 0]=\int_{g(X) \leq 0} f_{X}(x) d x=P\left[T_{r}<t\right] \cong \frac{\Pi[g(X) \leq 0]}{\Pi_{\text {total }}}
$$


where $g \leq 0$ represents the 'failure' domain and $f_{X}(x)$ is the (unknown) joint distribution of the random variables entering the problem and the integral is evaluated over the failure domain. In Monte Carlo simulation, the failure probability can be approximately evaluated as the ratio of $\Pi[g(X) \leq 0]$, which is the number of samples satisfying the event in brackets, over $\Pi_{\text {total, }}$ which is the total number of samples generated in the simulations. In this paper, the time-varying fatigue failure probability is estimated using Monte Carlo simulation with $10^{6}$ samples, in accordance with a convergence study (Imam 2006).

\subsection{System modelling}

The connections in riveted bridges consist of a number of components; failure of an individual component may not lead to total connection failure. For example, a typical stringer-to-cross-girder connection, which is the focus of this investigation, consists of 4 angle clips and 10 rivets as shown in Figure 4. The approach described in Section 3.3 can be used to estimate the probability of fatigue failure of individual hot-spots on various connection components, e.g. of a single rivet or of a single hole etc. These individual probabilities of failure can then be combined using a system reliability approach to estimate the overall reliability of the connection.

The connection can be considered as a system consisting of 4 sub-systems, each representing a single angle clip. Each angle clip, in turn, consists of various hotspots as its individual elements (e.g. holes, rivets, fillets). For example, it can be seen in Figure 4(a) that there are three holes and three rivets on the leg of each angle clip that is connected to the cross-girder web (rivets/holes 1-3), whereas there are two holes and two rivets on its leg connected to the stringer web (rivets/holes 4-5). Furthermore, two hot-spots are considered along the fillet depth (fillets 1-2) 
Combined failure in hot-spots will result in angle clip failures whereas combined angle clip failures will in turn imply total connection failure.
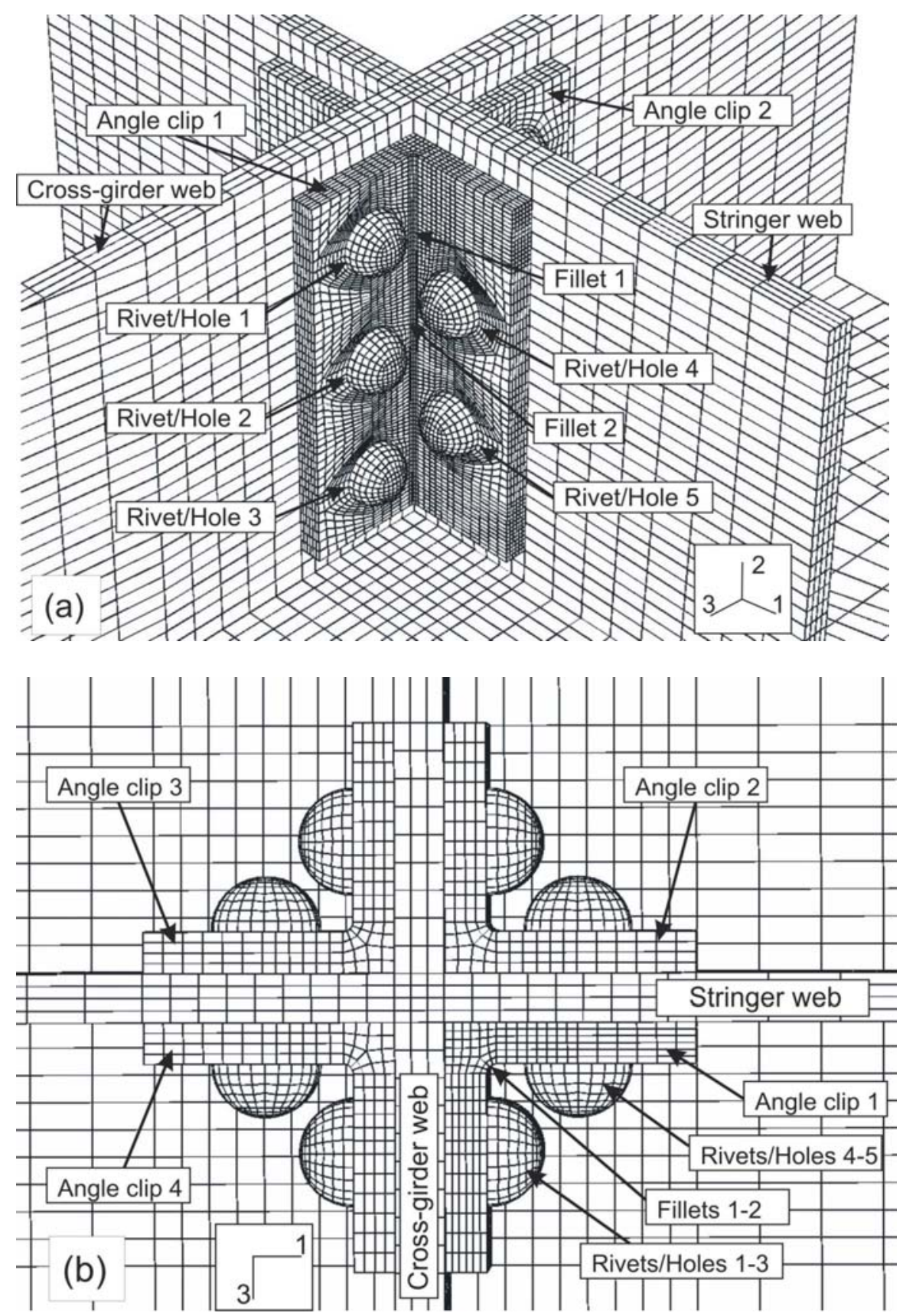

Figure 4. (a) Close-up view of the connection showing individual components (hotspots) (b) Cross-sectional view of connection showing individual angle clips. 
It is assumed that an individual angle clip can fail by one of the following five potential failure modes:

- Mode 1: failure of all three holes (holes 1-3) on the cross-girder leg of the angle clip

- Mode 2: failure of both holes (holes 4-5) on the stringer leg of the angle clip

- Mode 3: failure of all three rivets (rivets 1-3) on the cross-girder leg of the angle clip

- Mode 4: failure of both rivets (rivets 4-5) on the stringer leg of the angle clip - Mode 5: failure of both hot-spots along the depth of the angle fillet (fillets 1-2)

The probability of failure of each of these five modes can be estimated from the probabilities of failure of the individual hot-spots that contribute towards its failure (i.e. holes, rivets, fillets). For instance, since failure in mode 1 is assumed to take place when all three holes 1-3 have failed, this can be considered as a parallel sub-system with the holes 1-3 being its individual elements. Thus,

$$
P[\text { Mode1 }]=P[\text { Hole } 1 \cap \text { Hole } 2 \bigcap \text { Hole3 }]
$$

where the events in brackets imply failure at the named hot-spots. Assuming that hotspot failures are statistically independent, the probability of mode 1 failure can be estimated as

$$
P_{f(\text { Model })}=P_{f(\text { Hole } 1)} \cdot P_{f(\text { Hole } 2)} \cdot P_{f(\text { Hole })}
$$

The failure probability in any one of the remaining modes can be estimated in a similar fashion. It is important to note that correlation between failure modes should be expected. Assuming statistical independence herein is a conservative assumption, i.e. it increases the probability associated with each mode of failure.

Since failure in any of the five modes given above postulates failure of an angle, an angle clip can be considered as a series system consisting of these individual 
elements contributing to each failure mode. A system representation of an angle is shown in Figure 5. Thus,

$$
P[\text { Angle }]=P[\text { Mode } 1 \bigcup \text { Mode2 } \bigcup \ldots \cup \text { Mode } 5]
$$

and the probability of failure of an angle clip will be given as

$$
P_{f(\text { Angle })}=1-\left\lfloor\left(1-P_{f(\text { Mode } 1)}\right) \cdot \ldots \cdot\left(1-P_{f(\text { Mode })}\right)\right\rfloor
$$

where each of the probabilities on the right hand side can be calculated in a similar manner to Equation (7).

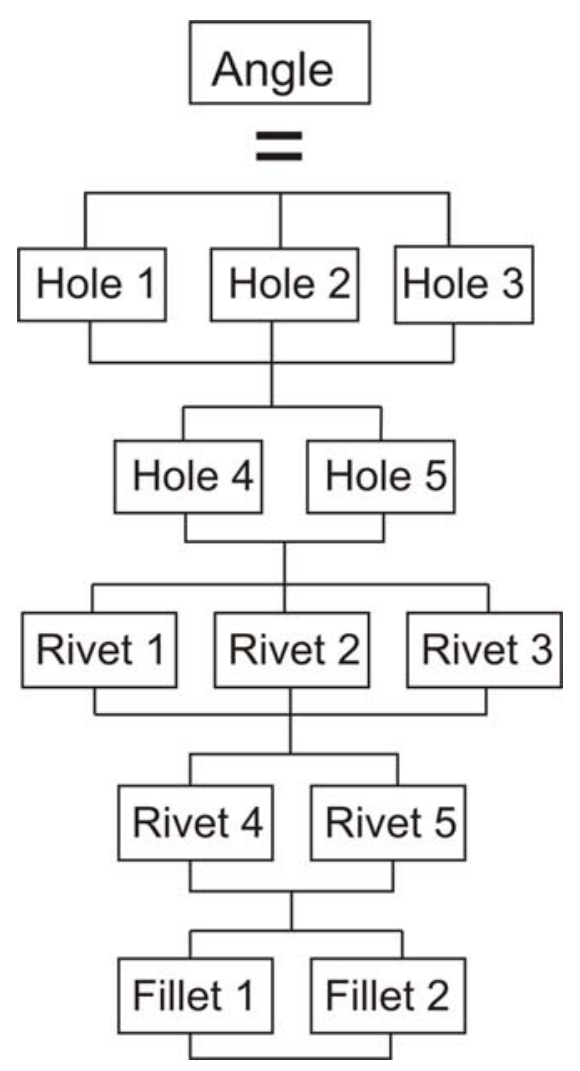

Figure 5. System representation of an angle clip sub-system.

Furthermore, the entire stringer-to-cross-girder connection can be considered as a parallel system comprised of angles as individual elements (Figure 6). A number of assumptions can be made regarding the number of angle failures that would lead to total connection failure. The probability of failure of the connection assuming failure 
of one angle only is given by Equation 9. Assuming that any two angle failures would lead to total connection failure, the system probability of failure can be calculated as $P[$ Connection $]=P\left[\begin{array}{l}(\text { Angle } 1 \text { and Angle2) or (Angle1 and Angle3) or (Angle1 and Angle4) } \\ \text { or (Angle2 and Angle3) or (Angle2 and Angle4) or (Angle3 and Angle4) }\end{array}\right]$

The bounds for the parallel system representation of the connection shown in Figure 6 can be expressed as

$$
P_{f(\text { Angle } 1)} \cdot P_{f(\text { Angle } 2)} \cdot P_{f(\text { Angle } 3)} \cdot P_{f(\text { Angle } 4)} \leq P_{f(\text { Connection })} \leq \min \left\lfloor P_{f(\text { Angle })}\right\rfloor
$$

The lower bound, i.e. $P_{f(\text { Angle })} \cdot \ldots \cdot P_{f(\text { Angle })}$, corresponds to statistically independent failure modes and is, therefore, not conservative. On the other hand, a conservative bound, which corresponds to the case of perfectly correlated modes, is, in this case, given by the upper bound, i.e. $\left.\min \left[P_{f(\text { Angle } i)}\right)\right]$.

For the purposes of this investigation, only the stresses at different hot-spots on angle clip 1 were obtained and post-processed using the TCD. The stresses on the remaining angle clips (2-4) are assumed to be equal to those in angle clip 1, which in reality is not necessarily true. Consequently, in this paper, the probability of failure of any angle is taken to be the same $\left(P_{f(\text { Angle })}=P_{f(\text { Angle })}=P_{f(\text { Angle })}=P_{f(\text { Angle } 4)}\right)$.

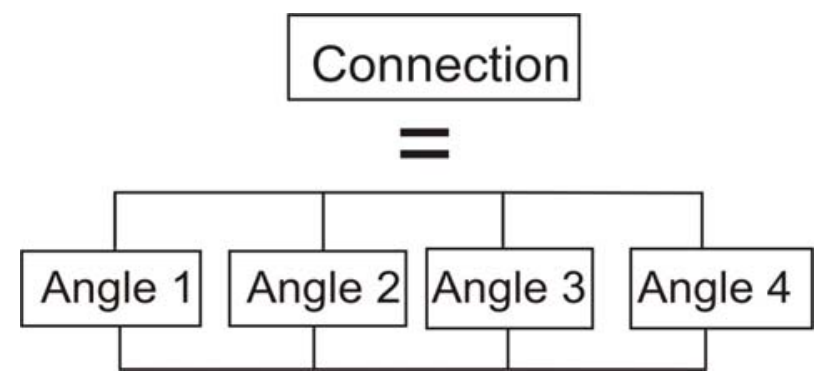

Figure 6. System representation of stringer-to-cross-girder connection. 


\subsection{Damage scenarios}

The clamping force developed in a rivet is difficult to control, as it is affected by a number of factors such as the stiffness of the rivet and the connected plates, the driving and finishing temperatures, the method employed for riveting (Al-Emrani 2002), as well as the skill of individual workers. Field measurements have shown that there is a wide variation in the magnitude of the clamping stress developed with values ranging between $30 \mathrm{MPa}$ and $280 \mathrm{MPa}$, lower values being observed in rivets driven in the field. This variation is observed not only for connections with different geometric properties and driving conditions but also in rivets taken from the same riveted connections. In order to quantify the effect of rivet clamping force on the fatigue reliability of the investigated stringer-to-cross-girder connection, the analyses are carried out by assuming two levels of clamping for all rivets, i.e. a low to moderate clamping stress of $100 \mathrm{MPa}$ and a high clamping stress of $200 \mathrm{MPa}$.

Rivet defects may also affect the fatigue performance of a riveted connection, as shown in an earlier deterministic study (Imam et al. 2007). In this paper, in addition to different rivet clamping stresses, the effect of a complete loss of rivet clamping force in each rivet is also investigated. Loss of clamping stress in a rivet may be gradual over a number of years through relaxation of the rivet due to vibrations and fretting between the connection components (Al-Emrani 1999). Moreover, tensile overloads in the rivets may also cause local yielding in the rivet head-to-shank junction leading to a partial or even complete loss of clamping force.

\section{Results and Discussion}

\subsection{Critical volumes for TCD}

As presented earlier, the TCD method is based on determining a critical distance which defines the region within which stress averaging is to be performed for fatigue damage calculations. The critical volumes at the different hot-spots of the connection 
under investigation have been determined in earlier studies (Righiniotis et al. 2008). For presentation purposes, some examples of these critical volumes, which are in the form of a hemisphere centred at the notch (stress concentration) and having a radius of $1.54 L$, are also shown here in Figures 7 and 8 . In this paper, the stresses are estimated using a fixed $L=1.73 \mathrm{~mm}$, as determined in section 3.2.1. Within the TCD context, maximum principal stresses are averaged within these critical volumes and the average maximum principal stress history is determined for each train that passes over the bridge. The rainflow counting method is used to convert these stress histories into stress range blocks which are then updated, within a probabilistic framework (as described in Section 3) through Monte Carlo simulations, to generate annual response spectra for each hot-spot within the connection.
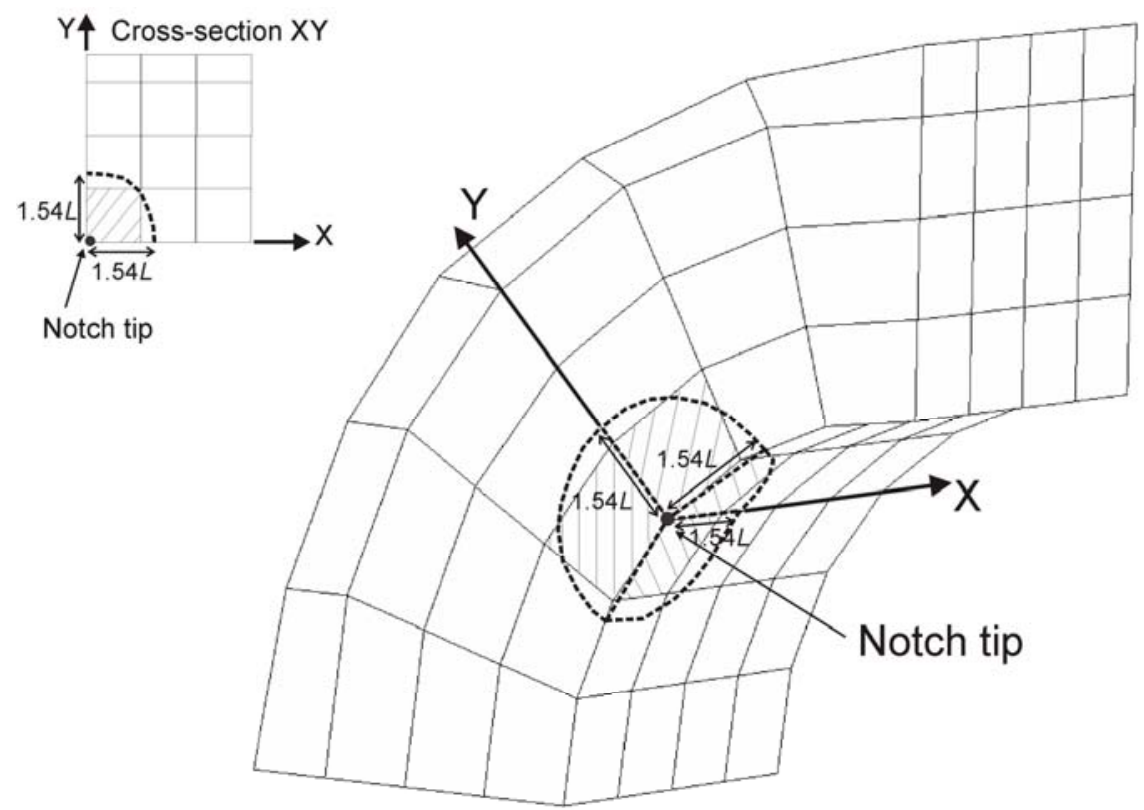

Figure 7. Critical volume around a hole perimeter. 

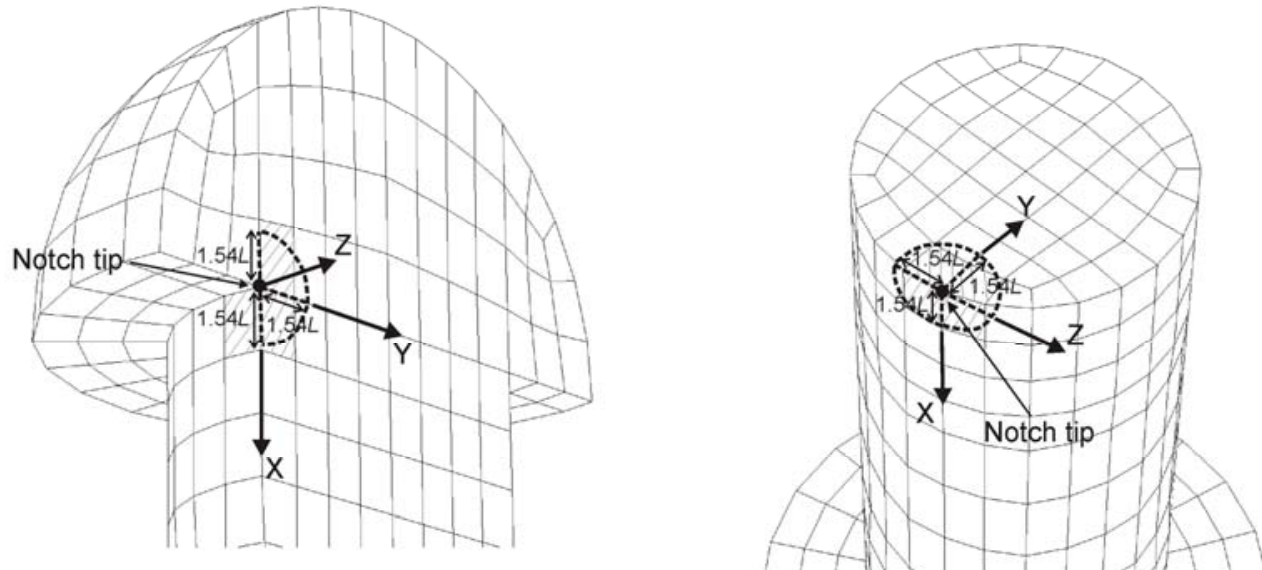

Figure 8. Critical volume around the rivet perimeter at its head-to-shank intersection.

\subsection{Fatigue load spectra}

Figures 9 and 10 show typical probabilistic fatigue load spectra generated for hotspots on the angle fillet 1 and hole 3 of the connection, respectively (see Figure 4(a)). The corresponding means and standard deviations of the stress range are also depicted in the figures. As mentioned previously, the spectra include the random effect of $D A F, \alpha$ and $f_{t j}$. The results pertain to the case of a $200 \mathrm{MPa}$ clamping stress in all the rivets of the connection.

It is evident that most stress cycles experienced by these particular hot-spots are below the mean fatigue limit of the material $(183 \mathrm{MPa})$. This was also observed in the fatigue load spectra developed in earlier studies using nominal, instead of local, stresses (Imam et al. 2008). Probability distributions may also be fitted to the fatigue load spectra as was done in the past by the authors in the case of the S-N nominal stress analysis of the connection (Imam et al. 2008). The challenge in using analytical distributions is to capture accurately the stress ranges which are close to the fatigue limit (right tail of the histograms in Figures 9 and 10) since these give the highest contribution to the overall fatigue damage. 


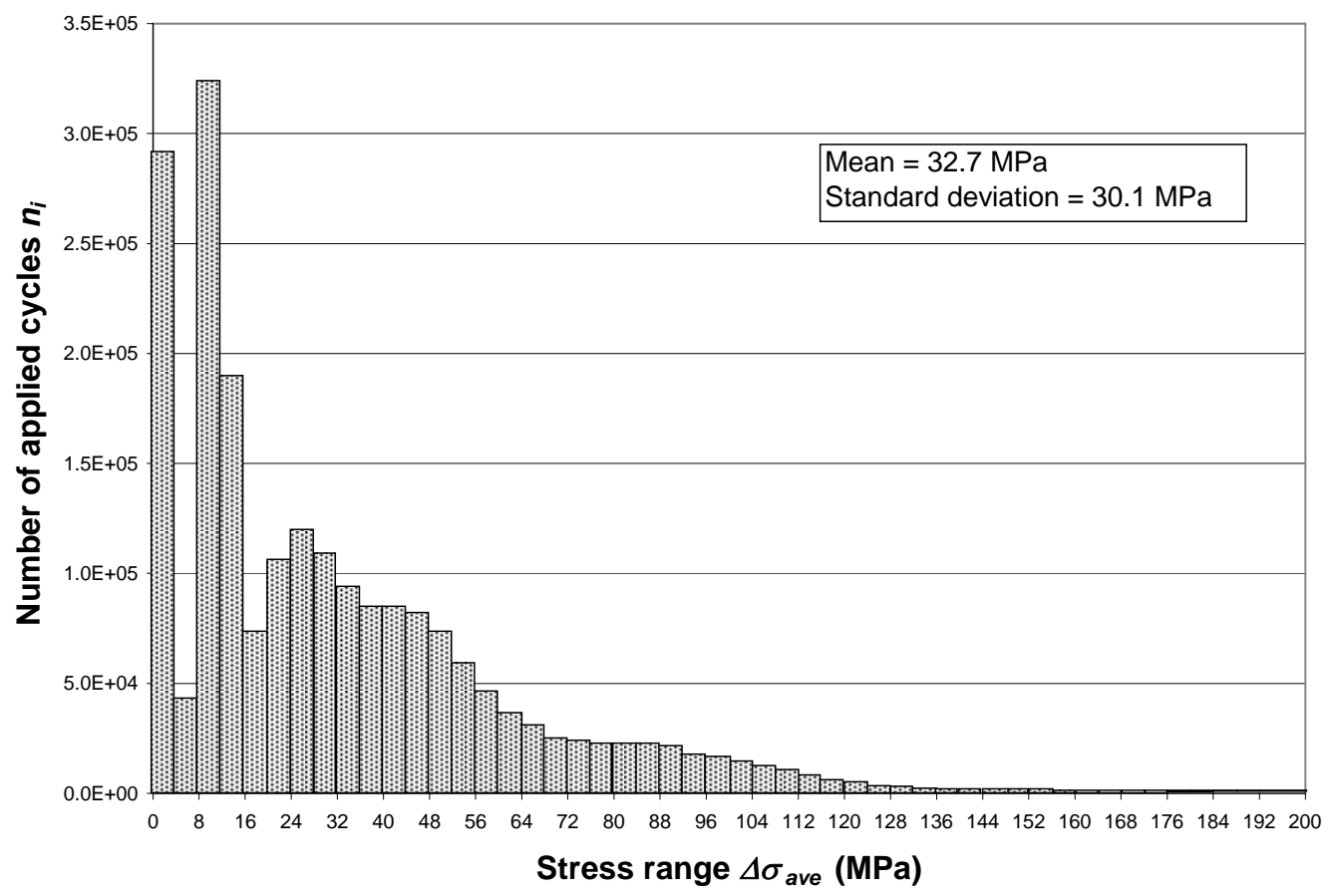

Figure 9. Annual fatigue load spectrum for angle fillet 1 (rivet clamping stress $=200$ $\mathrm{MPa})$.

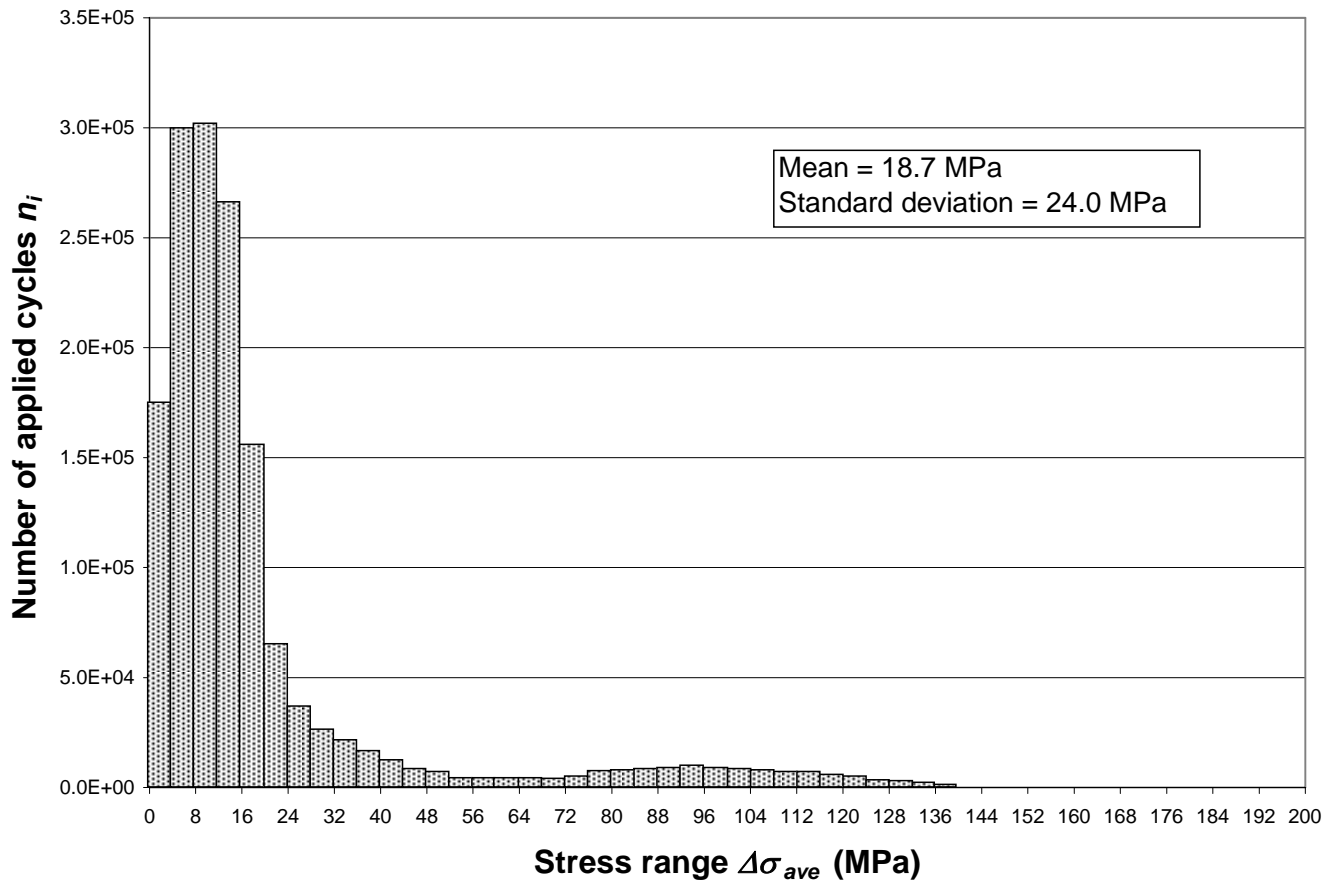

Figure 10. Annual fatigue load spectrum for hole 3 (rivet clamping stress $=200 \mathrm{MPa}$ ). 
Table 3 summarises the first and second order moment statistics for the annual fatigue load spectra of each connection hot-spot and for the two values of rivet clamping stress (100 and $200 \mathrm{MPa})$. The mean stress range at a detail can be considered as an important parameter in terms of fatigue reliability estimation and fatigue assessment. It can be seen that a higher clamping force in the rivets is beneficial in terms of reducing the mean stress range experienced by the majority of the hot spots in the connection. The exception is at the angle fillets where it can be seen that an increase in rivet clamping force results in increase in their mean stress range which can be attributed to the higher restraint experienced by the angle clip.

\begin{tabular}{|c|c|c|c|c|}
\hline \multirow{2}{*}{$\begin{array}{c}\text { Connection } \\
\text { hot-spot }\end{array}$} & \multicolumn{2}{|c|}{ Clamping stress = 100 MPa } & \multicolumn{2}{c|}{ Clamping stress =200 MPa } \\
\cline { 2 - 5 } & Mean (MPa) & $\begin{array}{c}\text { Standard } \\
\text { deviation (MPa) }\end{array}$ & Mean (MPa) & $\begin{array}{c}\text { Standard } \\
\text { deviation (MPa) }\end{array}$ \\
\hline Hole 1 & 30.0 & 24.3 & 17.2 & 18.3 \\
\hline Hole 2 & 32.4 & 21.9 & 25.2 & 18.1 \\
\hline Hole 3 & 22.0 & 25.8 & 18.7 & 24.0 \\
\hline Hole 4 & 38.4 & 27.4 & 27.9 & 24.0 \\
\hline Hole 5 & 43.9 & 34.6 & 35.1 & 31.8 \\
\hline Rivet 1 & 36.8 & 28.2 & 32.1 & 20.2 \\
\hline Rivet 2 & 35.2 & 28.1 & 28.6 & 22.4 \\
\hline Rivet 3 & 40.8 & 32.6 & 31.8 & 27.2 \\
\hline Rivet 4 & 31.6 & 24.3 & 26.4 & 21.5 \\
\hline Rivet 5 & 28.7 & 23.3 & 27.8 & 19.5 \\
\hline Fillet 1 & 30.2 & 28.3 & 32.7 & 30.1 \\
\hline Fillet 2 & 28.3 & 26.5 & 29.8 & 26.9 \\
\hline
\end{tabular}

Table 3. First and second order statistics for stress range spectra at hot-spots

\subsection{Fatigue reliability}

The fatigue damage in each hot-spot is calculated from its annual fatigue load spectrum, similar to Figures 9 and 10. The probability of failure and the remaining fatigue life are, then, estimated through Monte Carlo simulations using Equations 5 and 4 , respectively. 
Figures 11 and 12 show the probability of fatigue failure from year 2010 onwards for the different connection hot-spots shown in Figure 4(a). The results in Figure 11 are presented for a $100 \mathrm{MPa}$ clamping stress in all rivets whereas Figure 12 is for the case of $200 \mathrm{MPa}$ clamping stress. As mentioned earlier, these hot-spots are then treated as the individual elements of a structural system representing an angle clip. The probability of failure of the system, comprising of one angle only and calculated using Equation 9, is also shown in these figures for comparison purposes.

It is evident from Figures 11 and 12 that there is a very wide range in the probabilities of failure of the different hot-spots. This overall range appears to be narrower in the case of the lower rivet clamping stress of $100 \mathrm{MPa}$ where the failure probabilities are generally higher as compared to the case of $200 \mathrm{MPa}$ clamping stress. The remaining fatigue lives of the hot-spots range, for a $2.3 \%$ (design) probability of failure, from a couple of years up to $>100$ years. This illustrates the localised nature of the fatigue phenomenon with the damage being initiated from specific stress concentrations within a connection.

An increase in rivet clamping force can be seen to decrease the probability of failure for all holes and all rivets of the connection. Although higher clamping results in higher initial stresses being present in the rivets and, therefore higher stress ratio $\left(\sigma_{\min } / \sigma_{\max }\right)$ values, it reduces considerably the stress ranges experienced as a result of train loading, thus resulting in an overall reduction in fatigue damage accumulation. Different stress ratios would result in an upward or downward shift of the S-N curve of the material, however, in this paper, the effect of the stress ratio is neglected. Higher clamping in the rivet also affects the stresses experienced around the holes by introducing compression which has a beneficial effect on reducing the overall stress ranges at the hot-spots. 


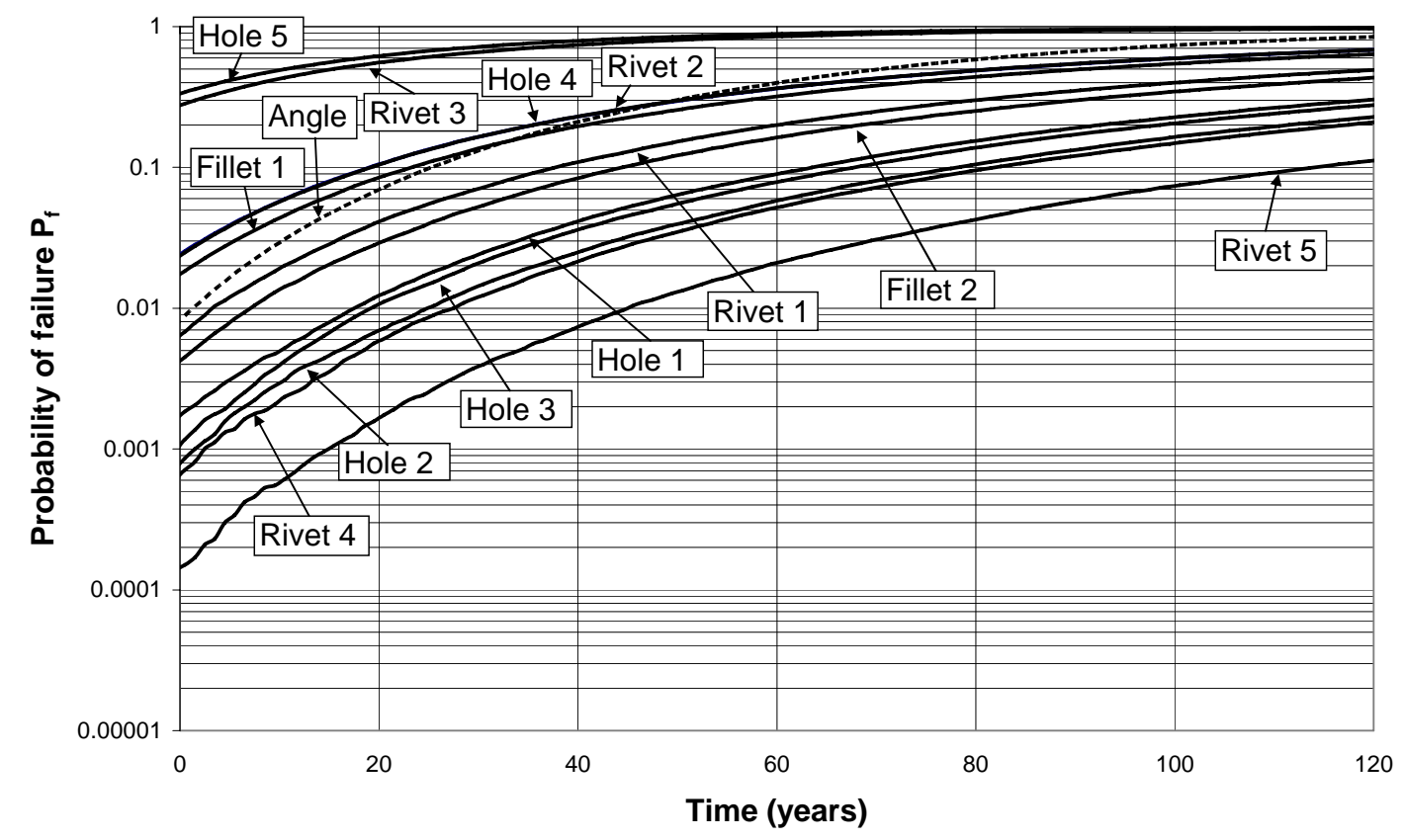

Figure 11. Probability of fatigue failure versus time for different connection hot-spots and angle system (rivet clamping stress $=100 \mathrm{MPa}$ ).

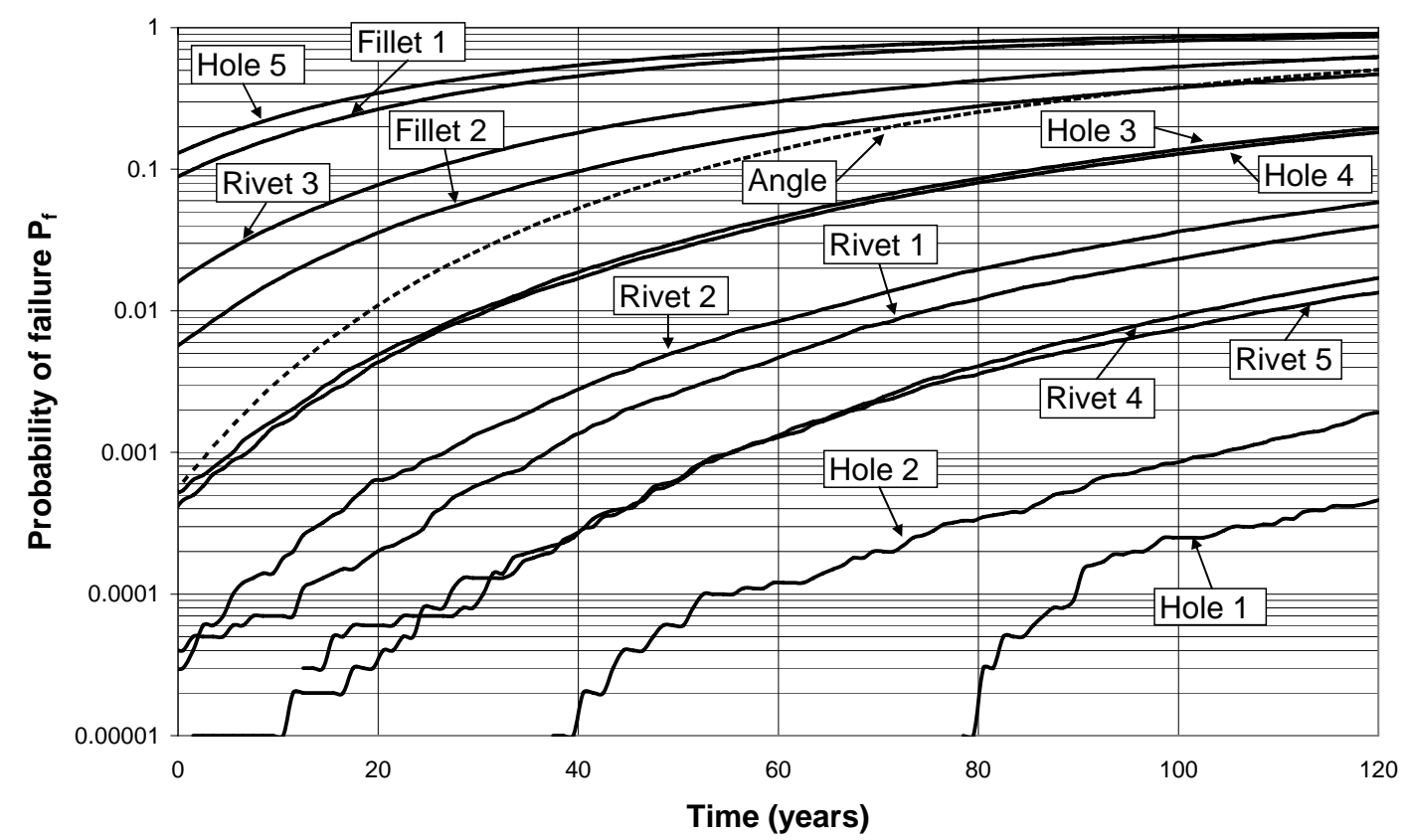

Figure 12. Probability of fatigue failure versus time for different connection hot-spots and angle system (rivet clamping stress $=200 \mathrm{MPa}$ ). 
It can also be seen from the Figures 11 and 12 that the fatigue criticality ranking of the hot-spots is affected by the magnitude of the rivet clamping force. However, the highest failure probability (lowest remaining life) for both values of clamping force is obtained for hole 5 (see Figure 4(a)) which is found to be the most critical location in the connection. The figures also show that a higher rivet clamping stress has an adverse effect on the fatigue reliability of the angle fillets 1 and 2 due to the higher restraint provided to the connection and accompanying larger stresses and associated stress ranges (see also Table 3). Therefore, in high clamping connections, fatigue cracking may initiate either from hole 5 or from the fillet area near the top side of the angle clip. Such type of cracking has been observed in the field in damaged stringer-to-cross-girder connections (Al-Emrani 2002).

The system failure probabilities, assuming one-angle, two-angle, and fourangle systems, calculated through Equation (11), are presented in Figure 13 for the two assumed clamping stress values. As mentioned earlier, the probability of failure for a one-angle system model represents the upper bound solution (right hand side of Equation (11) whereas the two- and four-angle models represent the lower bound solution (left hand side of Equation (11)). On the same figure, the reliability profiles obtained in earlier studies by the authors using the nominal (S-N) stress method (Imam et al. 2008) and modelling the connection as a single component are also shown. The latter had been obtained for different fatigue detail S-N classifications according to British [Class Wrought Iron (Railtrack 2001), Modified Class B (BS5400 1980)] and American (AREA (1996) punched holes) bridge design/assessment codes. Class Wrought Iron refers to riveted wrought-iron connections at locations of rivets whereas the S-N curve of Modified Class B has been shown to provide a good fit to available experimental fatigue results on riveted members (Imam 2006, Imam et al. 
2008). The AREA punched holes classification refers to riveted details having normal clamping force and assembled by punching holes.

It can be seen in Figure 13 that, irrespective of the rivet clamping stress, the failure probability of the connection (system) consisting of one angle only is considerably higher than the case of assuming a two- or a four-angle system. In terms of remaining fatigue life, for a $2.3 \%$ probability of failure and $200 \mathrm{MPa}$ clamping stress, a one-angle, two-angle and four-angle system results in 28, 63 and >100 years whereas in the case of $100 \mathrm{MPa}$ clamping stress in the rivets the remaining life obtained is 8, 33 and 60 years. This demonstrates the significant effect of rivet clamping force on the fatigue reliability of the connection system, with higher clamping stresses reducing the probability of system failure of the connection for all system modelling assumptions (1-, 2- and 4-angles).

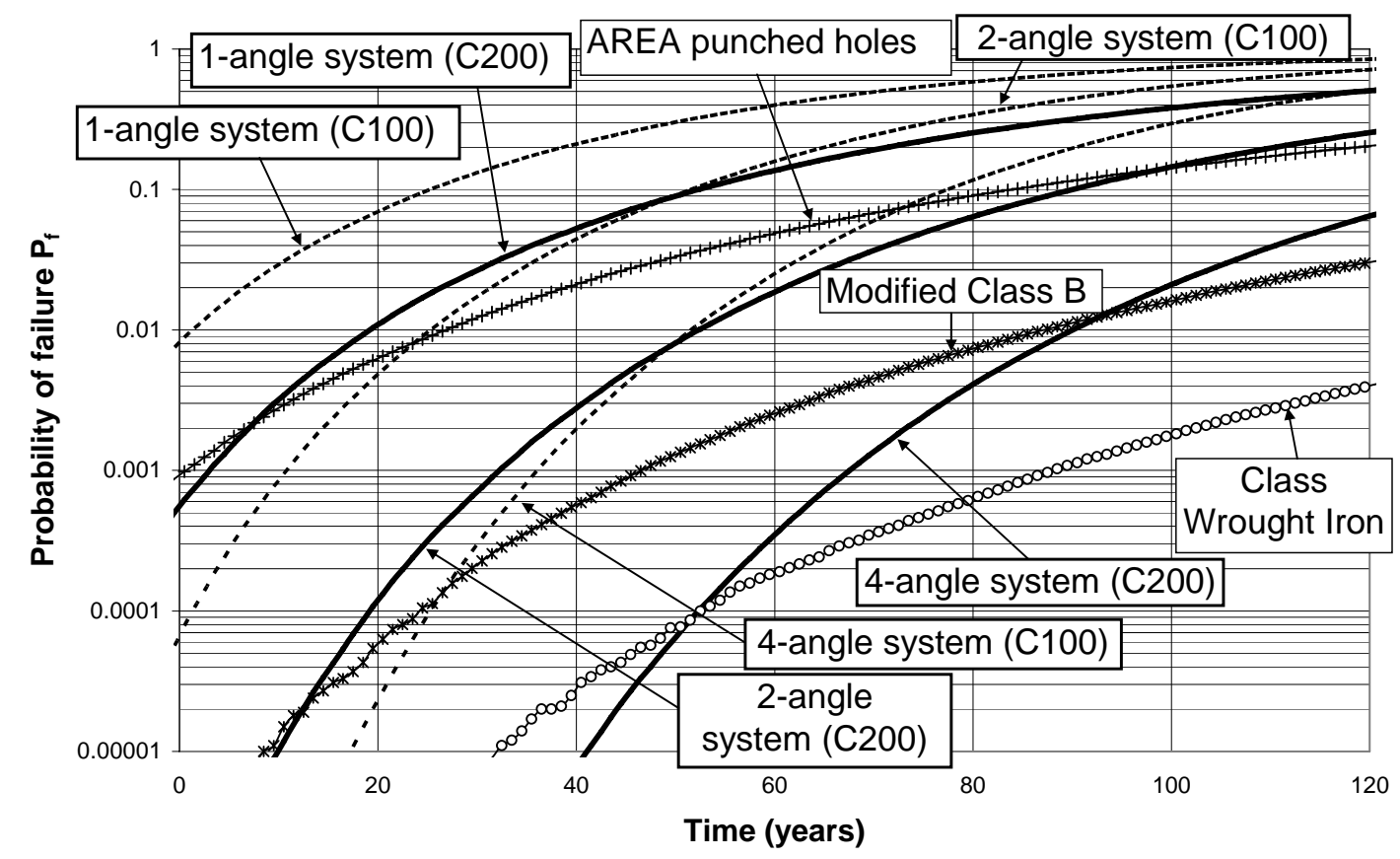

Figure 13. System probability of failure versus time obtained using the TCD for two rivet clamping stress values (100 $\mathrm{MPa}(\mathrm{C} 100)$ and $200 \mathrm{MPa}(\mathrm{C} 200))$ compared to the probability of failure obtained using the $\mathrm{S}-\mathrm{N}$ method (nominal stresses). 
By comparing the system reliability profiles with those obtained in previous studies through the traditional nominal stress (S-N) approach, it can be seen that a one-angle system assumption gives more conservative remaining life estimates than its nominal stress counterparts for both rivet clamping stress values. On the other hand, the remaining life estimates obtained from the four-angle system (for both clamping stress values) and the two-angle system (200 MPa clamping stress) can be seen to lie between its AREA (1996) punched holes and Class Wrought Iron (Railtrack 2001) counterparts. The TCD method employed in this paper appears to result in a more rapid increase in the probability of failure of the connection with time as compared to the nominal stress method.

\subsection{Damage scenarios}

The effect of the different damage scenarios, in terms of complete loss of each rivet clamping force, on the fatigue reliability of the connection is shown in Figures 14, 15 and 16 for one-angle, two-angle and four-angle system representation of the riveted connection, respectively. The probability of failure of the undamaged connection over time is also shown in the figures for comparison purposes. The results are presented for the case of having a $200 \mathrm{MPa}$ clamping stress in all the connection rivets.

It can be seen in Figures 14-16 that complete loss of the clamping force in rivet 3 has the most detrimental effect on the fatigue reliability of the connection. For a $2.3 \%$ probability of failure, loss of clamping in this rivet results in a 40 to $75 \%$ reduction in the remaining fatigue life, the highest reduction being observed in the case of a one-angle system representation of the connection. On the other hand, loss of clamping in rivet 1 results in a 10 to $30 \%$ reduction in the remaining fatigue life of the connection, which is notably lower than the first damage scenario. The damage scenarios associated with the other rivets (rivet 2, 4 and 5) were not found to be as 
highly critical as the previous cases resulting in only a small reduction in the fatigue reliability of the connection in the case of rivet 4 ; for rivets 2 and 5 , an increase in the overall connection reliability can be observed.

The results obtained in Figures 14-16 reveal that loss of clamping force in a connection rivet does not necessarily result in a detrimental effect on the fatigue reliability of the connection. For the particular stringer-to-cross-girder connection considered in this paper, loss of clamping force in the top and bottom rivets connecting the leg of the angle to the cross-girder web was found to affect the fatigue performance of the connection adversely. It is not, therefore, surprising that the majority of the fatigue damage cases observed in the field in similar riveted connections were concentrated in either the top or bottom rivets (Al-Emrani 1999, 2002).

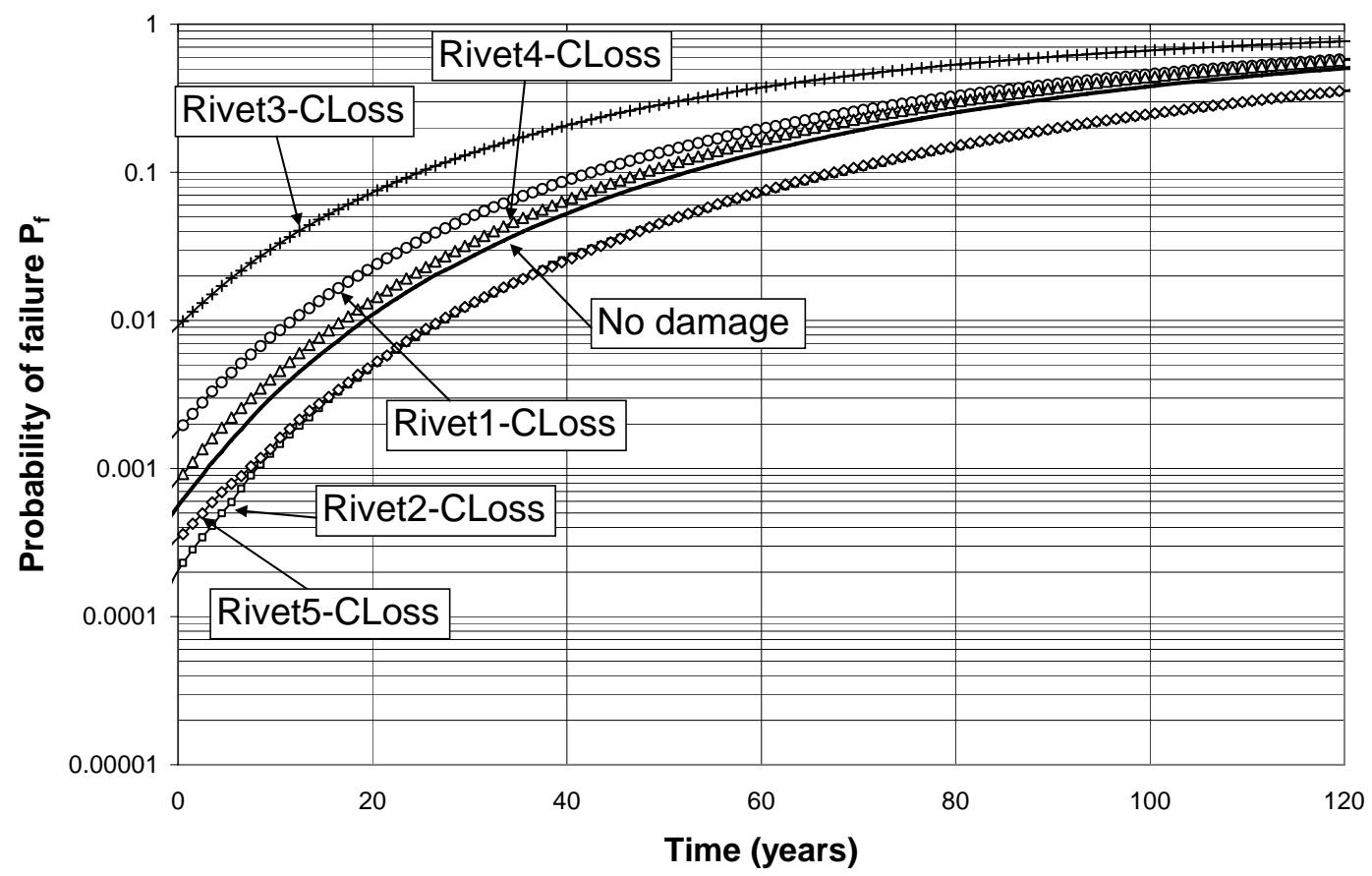

Figure 14. Probability of fatigue failure versus time for different rivet clamping force damage scenarios assuming a one-angle system (rivet clamping stress $=200 \mathrm{MPa}$ ). 


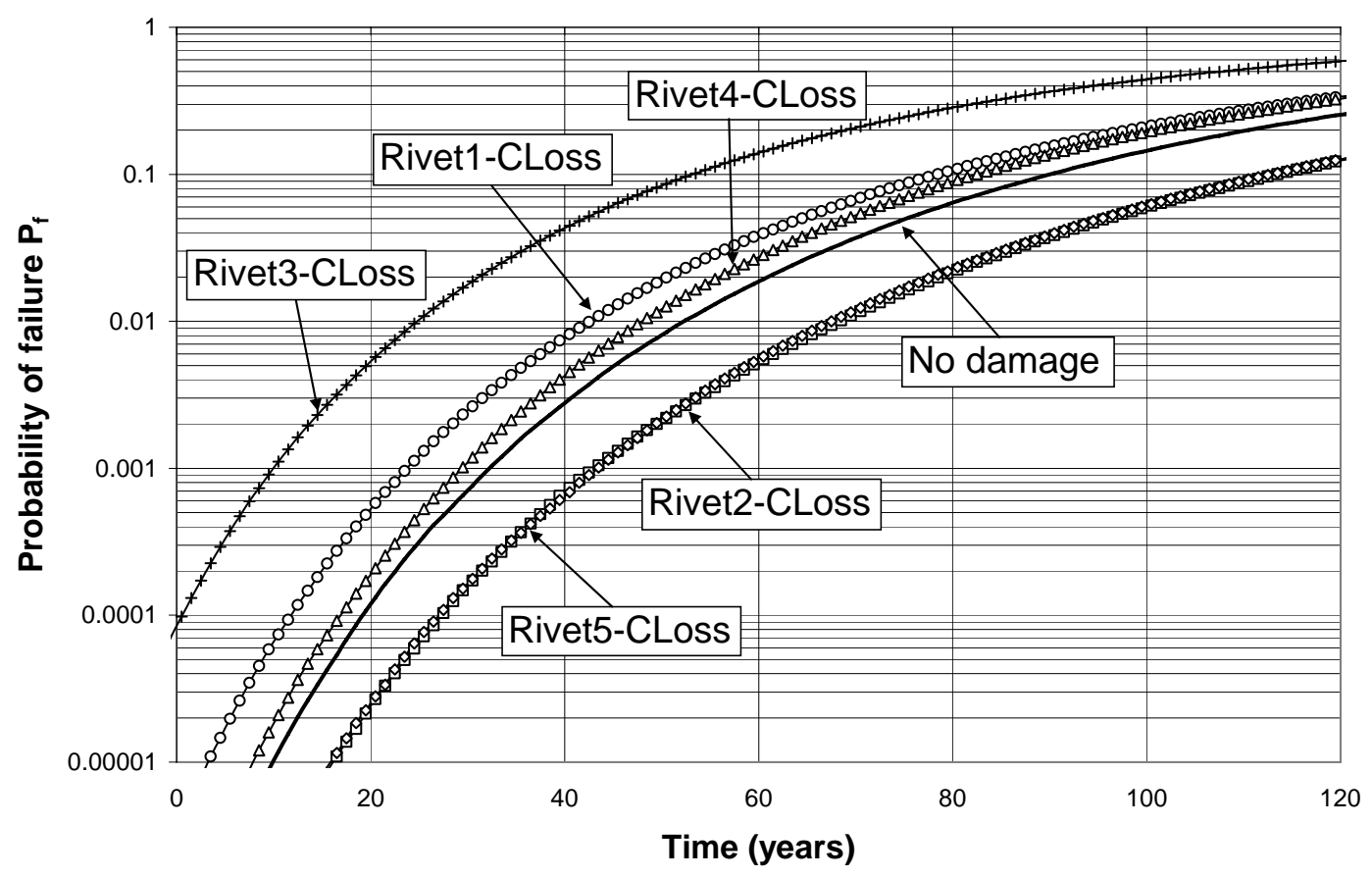

Figure 15. Probability of fatigue failure versus time for different rivet clamping force damage scenarios assuming a two-angle system (rivet clamping stress $=200 \mathrm{MPa}$ ).

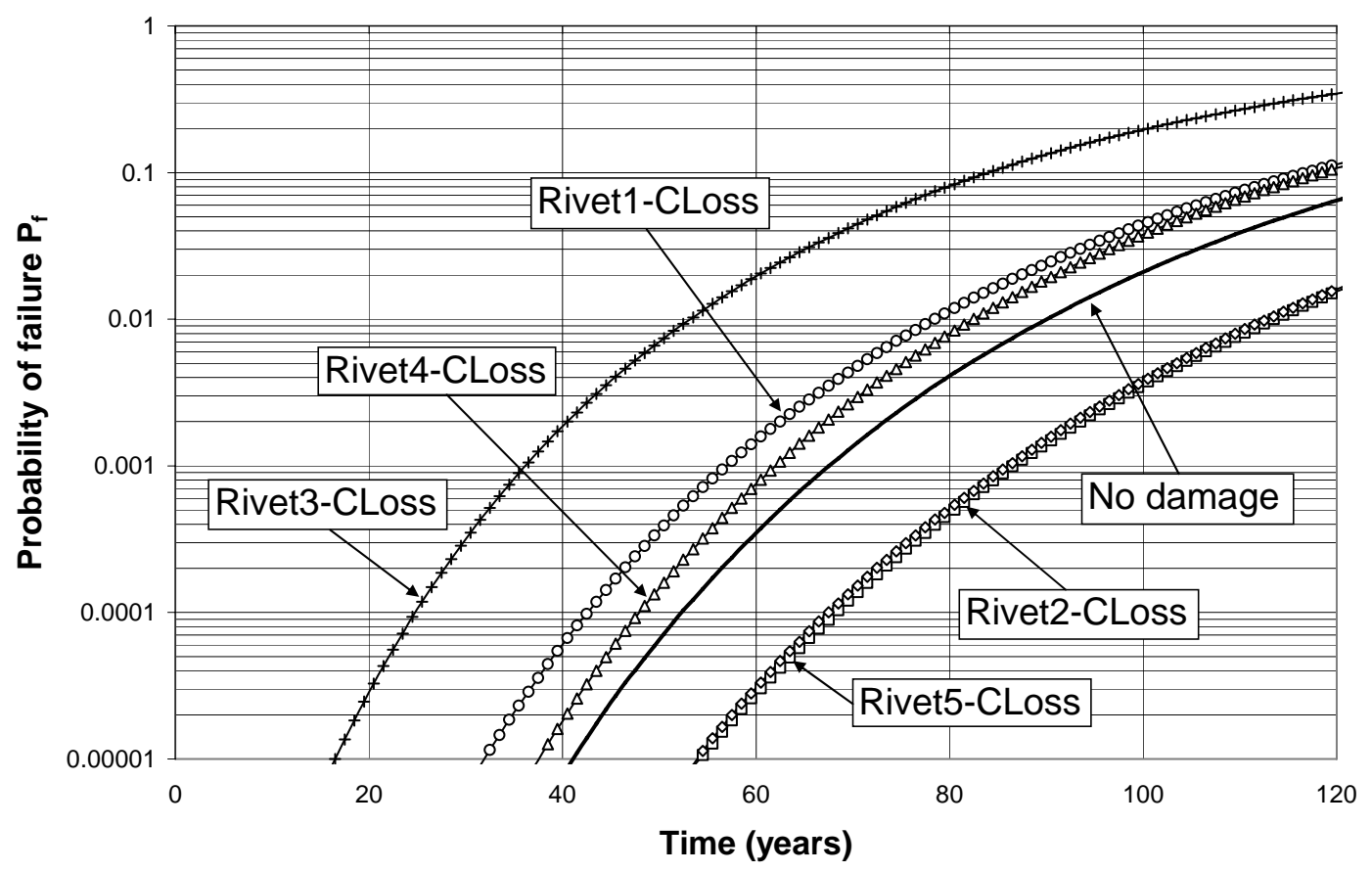

Figure 16. Probability of fatigue failure versus time for different rivet clamping force damage scenarios assuming a four-angle system (rivet clamping stress $=200 \mathrm{MPa}$ ). 
It should be noted that, for the purposes of the investigation, redistribution of stresses following a hot-spot (component) failure is not taken into account. The stresses on the remaining hot-spots are assumed to remain the same as in the undamaged state of the connection. In reality, the stresses will certainly change when a component has failed (e.g. a hole or a rivet) and the remaining hot-spots will carry the loads, which may lead to progressive failure of the connection. The current study has also adopted non-evolving loads from present day onwards, which is likely to be a non-conservative assumption given the current outlook on the development of rail freight in the UK and other countries. On the other hand, the assumption of independent failure modes in a weakest-link system, similar to the one investigated in this paper, leads to conservative results for the failure probability.

\section{Conclusions}

This paper presented a system-based model for fatigue assessment of riveted railway bridge connections building on previous work carried out by the authors which considered the connection as a single component. A finite element model of a typical, short-span, riveted railway bridge encompassing a detailed sub-model of a stringer-tocross-girder connection was used to convert the train loads to fatigue load effects. The criticality of the fatigue sensitive details (hot-spots) was assessed through the Theory of Critical Distances, a recently developed theory which considers the entire distribution ahead of any given stress concentration. Through Monte Carlo simulations, probabilistic fatigue load spectra for critical hot-spots on different components of the connection, such as holes, rivets, angle fillets were developed. These spectra showed that the majority of the stress cycles experienced by the hotspots are below the fatigue limit of the material. 
By using the fatigue load spectra developed, reliability profiles and probabilistic remaining fatigue life estimates for the connection were determined. Loading, resistance and modelling uncertainties were taken into account. On the loading side, the problem was randomised through dynamic amplification and the annual train frequencies. On the response side, the S-N curve and the cumulative damage model were treated as random. Finally, on the modelling side, the ratio between actual and calculated stresses was represented as a random variable. The riveted connection was treated through generic sub-systems capturing potential damage in identifiable hot-spots, such as rivets, holes and angle fillets. These hotspots were treated as the elements of a structural system susceptible to fatigue failure. The reliability profiles showed that the remaining life of the connection is sensitive to the assumptions made regarding the form of the system considered for the analysis. Low remaining lives were obtained for a number of the connection's hot-spots indicating that fatigue cracking may be imminent or may have already initiated in a number of similar existing bridge connections. Reliability profiles obtained from the TCD method and the system representation have shown a more rapid increase in failure probability of the connection compared to similar results obtained using the traditional S-N approach. The rivet clamping force was found to have a considerable effect on the reliability of the connection for all system representations. Loss of clamping force in the top or bottom rivets connecting the angle leg to the cross-girder web was found to result in a considerable reduction in the remaining fatigue life of the connection. Loss of clamping in the remaining rivets was found not to have a significant adverse effect on the connection's reliability.

It is unwise to generalise the results and conclusions presented in this paper to all types of riveted bridge connections with different configurations and geometries. 
For example, field observations from fatigue damage cases have shown that fatigue cracking occur more commonly at the top and bottom part of connections. This paper is a first attempt at presenting a general system-based representation appropriate for fatigue assessment of complex connections made up of a number of components. As discussed, further work is required so that it can serve as a useful tool in planning inspections and/or repair actions in similar riveted bridge connections.

\section{References}

Adamson, D.E., and Kulak, G.L., 1995. Fatigue tests of riveted bridge girders. Structural Engineering Report No 210. Department of Civil Engineering, University of Alberta, Edmonton, Canada.

Al-Emrani, M., 1999. Stringer-to-floor-beam connections in riveted railway bridges an introductory study of fatigue performance, Publ. S99:4. Chalmers University of Technology, Sweden.

Al-Emrani, M., 2002. Fatigue in riveted railway bridges - A study of the fatigue performance of riveted stringers and stringer-to-floor-beam connections. $\mathrm{PhD}$ Thesis. Chalmers University of Technology, Sweden.

American Association of State Highway Transportation Officials (AASHTO), 1998. LRFD bridge design specifications. $2^{\text {nd }}$ ed., Washington, DC, USA.

American Railway Engineering Association (AREA), 1996. Manual for railway engineering, Chapter 15: Steel Structures. Washington, DC, USA.

Bellett, D., Taylor, D., Marco, S., Mazzeo, E., Guillois, J., and Pircher, T., 2005. The fatigue behaviour of three-dimensional stress concentrations. International Journal of Fatigue, Vol 27 (3), 207-21.

BS5400, 1980. Steel, Concrete and Composite Bridges: Part 10: Code of Practice for Fatigue. London: British Standards Institute.

Byers, W.G., 1970. Impact from railway loading on steel girder spans. Journal of Structural Division (ASCE), Vol 96 (ST6), 1093-1103.

Byers, W.G., 1976. Rating and reliability of railway bridges. In: Proceedings of the National Structural Engineering Conference (ASCE), Vol. 1, Madison, 153170.

Cullimore, M.S.G., 1967. The fatigue strength of wrought iron after weathering in service. The Structural Engineer, Vol 45 (5), 193-199.

DNV, 2005. Fatigue design of offshore steel structures. Recommended Practice, DNV-RP-C203. Norway: Det Norske Veritas.

Doerk, O., Fricke, W., and Weissenborn, C., 2003. Comparison of different calculation methods for structural stresses at welded joints. International Journal of Fatigue, Vol 25 (5),359-369.

Dong, P., 2001. A structural stress definition and numerical implementation for fatigue analysis of welded joints. International Journal of Fatigue, Vol 23 (10), 865-876.

EC3, 2005. EN 1993-1-9: Eurocode 3: Design of steel structures - Part 1.9: Fatigue. CEN. 
Estes, A.C., and Frangopol, D.M., 1999. Repair optimization of highway bridges using system reliability approach. Journal of Structural Engineering (ASCE), Vol 125 (7), 766-775.

Ghosn, M., Moses, F., and Frangopol, D.M., 2010. Redundancy and robustness of highway bridge superstructures and substructures. Structure and Infrastructure Engineering, Vol 6 ( 1 \& 2), 257-278.

Helmerich, R., Kühn, B., and Nussbaumer, A., 2007. Assessment of existing steel structures. A guideline for estimation of the remaining fatigue life. Structure and Infrastructure Engineering, Vol 3 (3), 245-255.

Hendawi, S., and Frangopol, D.M., 1994. System reliability and redundancy in structural design and evaluation. Structural Safety, Vol 16 (1-2), 47-71.

Imam, B. 2006. Fatigue analysis of riveted railway bridges. $\mathrm{PhD}$ Thesis. University of Surrey, UK.

Imam, B., Righiniotis, T.D., Chryssanthopoulos, M.K., and Bell, B., 2006. Analytical fatigue assessment of a typical riveted UK rail bridge. Proceedings of the Institution of Civil Engineers (ICE) - Bridge Engineering, Vol 159 (3), 105116.

Imam, B.M., Righiniotis, T.D., and Chryssanthopoulos, M.K., 2007. Numerical modelling of riveted railway bridge connections for fatigue evaluation. Engineering Structures, Vol 29 (11), 3071-3081.

Imam, B.M., Righiniotis, T.D., and Chryssanthopoulos, M.K., 2008. Probabilistic fatigue evaluation of riveted railway bridges. Journal of Bridge Engineering (ASCE), Vol 13 (3), 237-244.

Melchers, R.E., 1999. Structural reliability analysis and prediction. Chichester: John Wiley \& Sons.

Micic, T., Chryssanthopoulos, M.K., and Baker M.J., 1995. Reliability analysis for highway bridge deck assessment, Structural Safety, Vol. 17, 135-150.

Miner, M.A., 1945. Cumulative damage in fatigue. Journal of Applied Mechanics, Vol 12 (3), 159-164.

Nowak, A.S., and Cho, T., 2007. Prediction of the combination of failure modes for an arch bridge system. Journal of Constructional Steel Research, Vol 63 (12), 1561-1569.

Okasha, N.M., and Frangopol, D.M., 2010. Time-variant redundancy of structural systems. Structural and Infrastructure Engineering, Vol 6 (1-2), 279-301.

Railtrack. 2001. RT/CE/C/025. Railtrack Line Code of Practice: The Structural Assessment of Underbridges. Railtrack, UK.

Righiniotis, T.D., Imam, B.M., and Chryssanthopoulos, M.K., 2008. Fatigue analysis of riveted railway bridge connections using the theory of critical distances. Engineering Structures, Vol 30 (10), 2707-2715.

Susmel, L., and Taylor, D., 2003. Fatigue design in the presence of stress concentrations. Journal of Strain Analysis for Engineering Design, Vol 38 (5), 443-452.

Sweeney, R.A.P., 1979. Importance of redundancy in bridge-fracture control. Transportation Research Record 711, Transportation Research Board, National Research Council, Washington DC, 23-30.

Sweeney, R.A.P., Oommen, G., and Le, H., 1997. Impact of site measurements on the evaluation of steel railway bridges. In: Report of IABSE Workshop, Lausanne: Evaluation of Existing Steel and Composite Bridges, Zurich, 139-147.

Taylor, D., 1999. Geometrical effects in fatigue: a unifying theoretical model. International Journal of Fatigue, Vol 21 (5), 413-420. 
Taylor, D., 2005. Analysis of fatigue failures in components using the theory of critical distances. Engineering Failure Analysis, Vol 12 (6), 906-914.

Taylor, D., 2007. The theory of critical distances: A new perspective in fracture mechanics. London: Elsevier.

Taylor, D., and Wang, G., 2000. The validation of some methods of notch fatigue analysis. Fatigue and Fracture of Engineering Materials and Structures, Vol 23 (5), 387-394.

Tobias, D.H., and Foutch, D.A., 1997. Reliability-based method for fatigue evaluation of railway bridges. Journal of Bridge Engineering (ASCE), Vol 2 (2), 53-60.

Wang, C.S., Chen, A.R., Chen, W.Z., and Xu, Y., 2006. Application of probabilistic fracture mechanics in evaluation of existing riveted bridges. Bridge Structures: Assessment, Design and Construction, Vol 2 (4), 223-232.

Wang C.S., Xu, Y., Chen, A.R., and Chen W.Z., 2007. System fatigue damage reliability assessment of railway riveted bridges. Key Engineering Materials, Vol 347, 173-178.

Wirsching, P.H., 1995. Probabilistic fatigue analysis. In: C. Sundararajan, ed. Probabilistic Structural Mechanics Handbook. New York: Chapman \& Hall. 